\title{
A Narrative Review of Body Mass Index in Pediatrics and its Relationship with Stability of Slipped Capital Femoral Epiphysis
}

\section{Phong Truong1*, Long Truong ${ }^{1}$, Kristina Kuklova ${ }^{2}$, Natalie Brush ${ }^{3}$, Luke Vincent $^{3}$ and Jonathan Schneider ${ }^{1}$}

${ }^{1}$ Department of Orthopedic Surgery, Larkin Community Hospital, South Miami, FL, USA

${ }^{2}$ American University of Antigua College of Medicine, St. Johns, Antigua and Barbuda

${ }^{3}$ University of Central Florida College of Medicine, Orlando, FL, USA

*Corresponding Author: Phong Truong, Department of Orthopedic Surgery, Larkin

Community Hospital, South Miami, FL, USA.
Received: August 24, 2020

Published: September 25, 2020

(C) All rights are reserved by Phong

Truong., et al.

\section{Abstract}

Purpose: To present a narrative review of the literature related to body mass index of children and adolescents and whether it relates to stability in slipped capital femoral epiphysis.

Method: A review of literature was conducted. Using the MEDLINE database and Google Scholar search engine, publications in the English language were queried in regards to body mass index in children as well as any effects of body mass index on stability of slips in slipped capital femoral epiphysis.

Results: Being overweight or obese is a risk factor for having slipped capital femoral epiphysis in adolescence; however, underweight or normal weight adolescent children have higher risk of unstable slips when compared to their overweight or obese peers. Perhaps bony changes in obese children play a protective role in decreasing risk of unstable slips. Epiphyseal cupping is a process in which the superior aspect of the capital femoral physis extends laterally towards the metaphysis. However, there has been no correlation shown between obesity and increased epiphyseal cupping.

Conclusion: This calls for more research related to possible structural changes in the proximal femur of obese adolescents that may play a role in protecting against unstable slips in slipped capital femoral epiphysis.

Keywords: Slipped Capital Femoral Epiphysis (SCFE); Body Mass Index; Orthopedic Surgery; Stability; Stable; Unstable

\section{Abbreviations}

SCFE: Slipped Capital Femoral Epiphysis; BMI: Body Mass Index; AVN: Avascular Necrosis; FAI: Femoroacetabular Impingement; CT: Computed Tomography

\section{Introduction}

Slipped capital femoral epiphysis (SCFE) is a common cause of hip pain in children under the age of 18 [1,2]. In SCFE, the femoral head sustains an injury, causing separation of the proximal femur physis at the hypertrophic cell zone in which the metaphysis translates in relation to the epiphysis [1,2]. This translation can be called a "slip", hence the name of the injury. The rate of SCFE differs from source to source, but it has been reported anywhere from 5.8 to 13.35 cases per 100,000 adolescents [3-5]. In some regions, the incidence is increasing; while in other regions it is decreasing. For example, there was a trend toward decreasing SCFE rates in Ontario between 2002 and 2011 [5]. On the contrary, there has been an increasing rate of SCFE in Korea since 1979, correlating with the increase in obesity in Korea during the same period [6]. The indig- 
enous population of southern Australia also shows an increase in both childhood obesity and rates of SCFE; in fact, the indigenous population is three times more likely to have SCFE than their nonindigenous counterparts [7].

Being overweight and obese is a risk factor for having SCFE during adolescence, as the literature widely supports [8-11]. Prevalence of overweight and obese children and adolescents in developed countries has increased; $23.8 \%$ of boys and $22.6 \%$ of girls were overweight or obese in 2013 [12]. With the rate of overweight and obese children continuing to rise, the fear is that SCFE rates may follow suit. SCFE can be categorized as stable or unstable; the risk of complications such as avascular necrosis is increased greatly in unstable slips $[3,13]$.

\section{Purpose of the Study}

The purpose of this article is to review the literature related to stable and unstable slips in SCFE, as well as find any correlation with overweight and obesity.

\section{Method}

A review of literature was conducted. Using the MEDLINE database and Google Scholar search engine, publications in the English language were queried in regards to $\mathrm{BMI}$ in children as well as any effects of BMI on stability of slips in SCFE. Example search queries included "body mass index SCFE" and "obesity SCFE unstable". Bibliographies of chosen studies were searched for additional sources. A total of 35 sources involving BMI in children, stability of SCFE, and BMI in SCFE stability were included in the narrative review.

\section{Results}

Body mass index in children

When discussing obesity and BMI in relation to SCFE, it is important to acknowledge the difference between BMI in adults and BMI in children. In adults, obesity is determined by a constant scale that is given by dividing a person's weight by their height squared. BMI of 18.5 to 24.99 is normal, 25.0 to 29.99 is overweight, and $30.0+$ is obese [14]. This is not how childhood obesity is calculated. In children and teens, age and sex is taken into consideration and is referred to as BMI-for-age. This BMI-for-age uses a percentile rather than a range of numbers to account for the differences in body composition between males and females as they age. This means that BMI in children are expressed relative to others of the same age and sex. For example, a BMI between the $85^{\text {th }}$ and $95^{\text {th }}$ percentile is considered overweight, while a BMI above the $95^{\text {th }}$ percentile is considered obese [15]. One study finds that BMI-for-age is superior to weight-for-height at overweight detection in children 3 to 19 years of age [16]. Another study uses weight, height, triceps skinfold and subscapular skinfold to estimate obesity in adolescents; it concludes that the different criteria are similar and highly specific for both sexes, but sensitivity for females is low [17].

\section{Stable versus unstable SCFE}

Determination of stability in SCFE is crucial as it lends appraisal to the risk of avascular necrosis (AVN) of the femoral head [13]. A SCFE is considered to be stable if a patient is able to bear weight on the affected extremity with or without the assistance of crutches. An unstable SCFE is when weight bearing is too painful for the patient even with the aid of crutches [18-20]. AVN has a greater rate of occurrence in SCFE with an unstable slip. The literature reports the rate of AVN in unstable SCFE to be $15 \%$ to $50 \%[1,18,21,22]$. The estimate of a recent meta-analysis goes as high as 9.4 times greater in unstable slips compared with stable slips [13].

The gold standard for treating SCFE is using in situ screw fixation, whether the slip is stable or unstable, as it has provided longterm function while minimizing complications [20,23,24]. Some providers treat stable SCFE with a single screw and unstable SCFE with two screws [25]. In unstable slips, one implicated cause of increased AVN is intracapsular hemorrhage causing increased intracapsular pressure and compressing critical blood vessels. Therefore, alternative treatment options are emerging to address that. Wright., et al. found that evacuating the hematoma followed by open reduction and fixation resulted in only a $5 \%$ rate of AVN in unstable slips [19].

\section{Body mass index and stability of SCFE}

It is widely accepted that being overweight or obese increases the risk of SCFE in adolescents, with many being above the $95^{\text {th }}$ percentile of BMI-for-age [9-11,26-28]. This may be due to the increased shear stresses on the physis [29]. However, there is relatively little data on the relationship between BMI and slip stability. In the literature search, two studies mentioned such a relationship. One study reported that patients without obesity were significantly more likely to present with an unstable SCFE compared to those who were obese. When stable SCFE is compared with unstable SCFE, the unstable slips have lower BMI [30]. This is supported by another recent article that reports underweight or normal weight patients are more likely to have unstable SCFE than their overweight or obese peers [31]. 
Morris., et al. mentioned that adolescents with cam morphology may develop extension of the capital femoral epiphysis in which the superior aspect of the physis extends laterally towards the metaphysis [32]. It may be an adaptive response to the shear stress on the physis and may confer physeal stability in SCFE, reducing the risk of unstable slips [33]. This physeal extension is referred to as epiphyseal cupping [34]. However, Hosseinzadeh., et al. reviewed pelvic computed tomography (CT) scans of 68 children and adolescents and found no correlation between obesity and increased epiphyseal cupping [35].

\section{Conclusion}

The current literature suggests that underweight or normal weight adolescent children have higher risk of unstable slips in cases of SCFE compared to their overweight or obese peers [30,31]. It is possible that bony changes, such as epiphyseal cupping, in the capital femoral epiphysis may play a protective role in decreasing unstable slips. However, this is not supported by evidence as there is no correlation between obesity and increased epiphyseal cupping [36]. We recommend further research related to structural changes in the proximal femur in obese adolescents and whether those changes are protective against unstable slips in SCFE.

\section{Conflicts of Interest}

We declare that we have no conflicts of interest in the authorship or publication of this work.

\section{Bibliography}

1. Mathew Smitha E and A Noelle Larson. "Natural History of Slipped Capital Femoral Epiphysis". Journal of Pediatric Orthopaedics 39.6 (2019): S23-S27.

2. Samelis Panagiotis V., et al. "Factors Affecting Outcomes of Slipped Capital Femoral Epiphysis". Cureus 12.2 (2020): 06883.

3. Vukasinović Zoran., et al. "Complications of Slipped Capital Femoral Epiphysis". Srpski Arhiv Za Celokupno Lekarstvo 135.1-2 (2007): 105-110.

4. Lehmann Charles L., et al. "The Epidemiology of Slipped Capital Femoral Epiphysis: An Update". Journal of Pediatric Orthopaedics 26.3 (2006): 286-290.

5. Ravinsky Robert., et al. "Epidemiology of Slipped Capital Femoral Epiphysis in Ontario, Canada”. Journal of Pediatric Orthopaedics 39.3 (2019): E165-E167.
6. Song Kwang Soon., et al. "Epidemiology and Demographics of Slipped Capital Femoral Epiphysis in Korea: A Multicenter Study by the Korean Pediatric Orthopedic Society". Journal of Pediatric Orthopaedics 29.7 (2009): 683-686.

7. AR Nguyen., et al. "Slipped Capital Femoral Epiphysis: Rising Rates with Obesity and Aboriginality in South Australia". The Journal of Bone and Joint Surgery British 93 (2011): 14161423.

8. Loder RT. "The Demographics of Slipped Capital Femoral Epiphysis. An International Multicenter Study". Clinical Orthopaedics and Related Research 322 (1996): 8-27.

9. M Poussa., et al. "Body Mass Index and Slipped Capital Femoral Epiphysis". Journal of Pediatric Orthopaedics B 12 (2003): 369-371.

10. Bhatia Nitin N., et al. "Body Mass Index in Patients with Slipped Capital Femoral Epiphysis". Journal of Pediatric Orthopaedics 26.2 (2006): 197-199.

11. Manoff Eric M., et al. "Relationship between Body Mass Index and Slipped Capital Femoral Epiphysis". Journal of Pediatric Orthopaedics 25.6 (2005): 744-746.

12. Ng Marie., et al. "Global, Regional, and National Prevalence of Overweight and Obesity in Children and Adults during 19802013: A Systematic Analysis for the Global Burden of Disease Study 2013". The Lancet 384.9945 (2014): 766-781.

13. Tosounidis Theodoros., et al. "Prognostic Significance of Stability in Slipped Upper Femoral Epiphysis: A Systematic Review and Meta-Analysis". Journal of Pediatrics 157.4 (2010): 674-680.

14. Yan Lijing L., et al. "BMI and Health-Related Quality of Life in Adults 65 Years and Older". Obesity Research 12.1 (2004): 6976.

15. Defining Childhood Obesity | Overweight and Obesity | CDC (2020).

16. Z Mei., et al. "Validity of Body Mass Index Compared with Other Body-Composition Screening Indexes for the Assessment of Body Fatness in Children and Adolescents". The American Journal of Clinical Nutrition 75 (2002): 978-985.

17. JC Farias Junior., et al. "Sensitivity and Specificity of Criteria for Classifying Body Mass Index in Adolescents". Revista de Saúde Pública 43 (2009): 53-59. 
18. RT Loder., et al. "Acute Slipped Capital Femoral Epiphysis: The Importance of Physeal Stability". Journal of Bone and Joint Surgery American 75 (1993): 1134-1140.

19. Wright Jonathan and Manoj Ramachandran. "Slipped Capital Femoral Epiphysis: The European Perspective". Journal of Pediatric Orthopedics 38 (2018): 1-4.

20. DD Aronsson., et al. "Slipped Capital Femoral Epiphysis: Current Concepts". The Journal of the American Academy of Orthopaedic Surgeons 14 (2006): 666-679.

21. David D Aronsson., et al. "Stable Slipped Capital Femoral Epiphysis: Evaluation and Management". The Journal of the American Academy of Orthopaedic Surgeons 4.4 (1996): 173181.

22. DW Boyer., et al. "Slipped Capital Femoral Epiphysis. LongTerm Follow-up Study of One Hundred and Twenty-One Patients". Journal of Bone and Joint Surgery American 63 (1981): 85-95.

23. Loder Randall T., et al. "Slipped Capital Femoral Epiphysis". Instructional Course Lectures 57 (2008): 473-498.

24. Wensaas Anders., et al. "Long-Term Outcome of Slipped Capital Femoral Epiphysis: A 38-Year Follow-up of 66 Patients". Journal of Children's Orthopaedics 5.2 (2011): 75-82.

25. Lang Pamela., et al. "The Outcome of In-Situ Fixation of Unstable Slipped Capital Femoral Epiphysis". Journal of Pediatric Orthopaedics Part B 28.5 (2019): 452-457.

26. Alghamdi Anas A., et al. "Slipped Capital Femoral Epiphysis and Primary Hyperparathyroidism: A Case Report". Clinical Medicine Insights: Endocrinology and Diabetes 9 (2016).

27. Song Hae Ryong., et al. "Unstable Slipped Capital Femoral Epiphysis: Reduction by Gradual Distraction with External Fixator. A Case Report". Journal of Pediatric Orthopaedics Part B 14.6 (2005): 426-428.

28. Kushare Indranil., et al. "Acute, Unstable Slipped Capital Femoral Epiphysis with Associated Congenital Coxa Vara". Journal of Pediatric Orthopaedics Part B 24.6 (2015): 511-514.

29. Zupanc Oskar., et al. "Shear Stress in Epiphyseal Growth Plate Is a Risk Factor for Slipped Capital Femoral Epiphysis". Journal of Pediatric Orthopaedics 28.4 (2008): 444-451.

30. Obana Kyle K., et al. "Slipped Capital Femoral Epiphysis in Children without Obesity". Journal of Pediatrics 218 (2020): 192-197.
31. Goldstein Rachel Y., et al. "The 'Skinny' SCFE”. Pediatrics Meeting Abstract, American Academy of Pediatrics (AAP) 140.1 (2017): 107-107.

32. Morris William Z., et al. "Capital Femoral Growth Plate Extension Predicts Cam Morphology in a Longitudinal Radiographic Study". Journal of Bone and Joint Surgery - American 98.10 (2016): 805-812.

33. Morris William Z., et al. "Capital Femoral Epiphyseal Extension May Confer Physeal Stability in Slipped Capital Femoral Epiphysis". Journal of Pediatric Orthopaedics 39.3 (2019): 119-124.

34. Morris William Z., et al. "Capital Femoral Epiphyseal Cupping and Extension May Be Protective in Slipped Capital Femoral Epiphysis: A Dual-Center Matching Cohort Study". Journal of Pediatric Orthopedics 40.7 (2020): 334-339.

35. Hosseinzadeh Shayan., et al. "Increased Body Mass Index Percentile Is Associated with Decreased Epiphyseal Tubercle Size in Asymptomatic Children and Adolescents with Healthy Hips". Journal of Children's Orthopaedics 14.3 (2020): 167-174.

\section{Assets from publication with us}

- Prompt Acknowledgement after receiving the article

- Thorough Double blinded peer review

- Rapid Publication

- Issue of Publication Certificate

- High visibility of your Published work

Website: https://www.actascientific.com/

Submit Article: https://www.actascientific.com/submission.php

Email us: editor@actascientific.com

Contact us: +919182824667 